\title{
Failure of Centrally Placed Objects to Control the Firing Fields of Hippocampal Place Cells
}

\author{
Arnaud Cressant, ${ }^{1}$ Robert U. Muller, ${ }^{2}$ and Bruno Poucet ${ }^{1}$ \\ ${ }^{1}$ Center of Research for Cognitive Neuroscience, Centre National de la Recherche Scientifique, 13402 Marseille, Cedex \\ 20, France, and 2Department of Physiology, State University of New York, Brooklyn, New York 11203
}

Previous work has shown that the angular position of hippocampal place cell firing fields is accurately controlled by the position of a single white cue card attached to the wall of a recording cylinder: when the card is rotated, fields rotate equally. In this study, we asked whether similar control could be exerted by three-dimensional objects placed directly in the recording arena. In each of several conditions, the locations of the objects relative to each other and their distances from the cylinder wall were fixed. In Experiment 1, the objects were all near the center of the cylinder. In this condition, the angular position of firing fields could, in general, not be predicted from the angular position of the object set. When a white wall card was added to the object arrangement, the stimulus ensemble exerted nearly ideal control over angular firing position. Nevertheless, when the card was withdrawn, the objects still did not control field position. In Experiment 2, place cells were re- corded in the presence of two new arrangements of the same objects used in Experiment 1. In the "clustered objects" condition, the objects were placed next to each other, $10 \mathrm{~cm}$ from the wall. In the "objects-at-periphery" condition, the objects were put against the cylinder wall by equally increasing the distances among the objects. In both conditions, we found virtually ideal control by the objects over angular field position. These results indicate that the failure of stimulus control in Experiment 1 must be attributable to the arrangement of the objects and not to the nature of the objects themselves. Overall, the results are in line with behavioral studies that show that it is very difficult to teach rats to locate food relative to landmarks inside the behavioral arena.

Key words: dorsal hippocampus; unit recordings; place cells; spatial learning; spatial memory; rat
Along with other species, rats display remarkable navigational abilities, which are inferred to depend on the existence of a map-like representation of the spatial environment (Gallistel, 1990; Poucet, 1993). Previous research aimed at determining the mechanisms required to implement neural maps has focused on the role of the hippocampal formation (O'Keefe and Nadel, 1978). One line of evidence in favor of a role of the hippocampal formation in navigation is provided by lesion studies. Such work reveals that damage to the hippocampus or associated structures leads to severe deficits in the ability to learn a wide variety of spatial tasks (Morris et al., 1982, 1990; Kelsey and Landry, 1988; Skelton and McNamara, 1992; Taube et al., 1992).

The strongest evidence for involvement of the hippocampal formation in processing spatial information is the existence of "place cells" (O'Keefe and Dostrovsky, 1971). Place cells are hippocampal pyramidal cells, the firing of which is strongly correlated with the rat's head position. Each place cell is characterized by a spatially delimited "firing field"; the cell fires rapidly when the rat's head is inside its field and is almost silent elsewhere

Received Sept. 17, 1996; revised Jan. 13, 1997; accepted Jan. 23, 1997.

Support for this work was provided by the Centre National de la Recherche Scientifique (CNRS) and by CNRS/National Science Foundation Grant 96/0690, NATO Grant CRG 940777, and U.S. Public Health Service-National Institutes of Health Grant 20686. We thank B. Arnaud and E. S. Hawley for help in constructing the unit recording system, L. Eberle and R. Fayolle for electronics, and S. Benhamou for helpful discussions. Portions of this work were presented in poster form at the 1995 and 1996 meetings of the Society for Neuroscience.

Correspondence should be addressed to Dr. Bruno Poucet, Center of Research for Cognitive Neuroscience, Centre National de la Recherche Scientifique, 31 Chemin Joseph-Aiguier, 13402 Marseille, Cedex 20, France.

Copyright (C) 1997 Society for Neuroscience $\quad 0270-6474 / 97 / 172531-12 \$ 05.00 / 0$ in the environment. The firing field may be anywhere in the environment.

A basic question about place cells is whether their firing fields are stable. In the simplest sense of stability, we mean that the field of a cell is the same when it is recorded under identical conditions on two or more occasions. In the prototypical experiment, a rat is brought into an environment and recording goes on for a predetermined number of minutes; the recording interval is called a session. The rat now is removed from the apparatus and put into a different place (often the home cage). Later, the rat is returned to the apparatus and another session is done. The firing field is stable if the positional firing patterns in the first and second sessions (and possible subsequent sessions) are indistinguishable except for details such as minor variations in firing rate.

There is a great deal of evidence that firing fields can be stable if the environment is unchanged between sessions done days or weeks apart (Muller et al., 1987; Thompson and Best, 1990). Clearly, fields can be stable only if place cell discharge stays in register with one or more constant stimulus features of the environment. Presumably, the critical stimulus features can be identified by altering components of the stimulus configuration and seeing how firing fields are affected. An obvious experiment is to rotate a candidate stimulus or stimulus ensemble with respect to the laboratory frame by a known number of degrees and do a new recording session. If the stimulus rotation reliably causes equal field rotations, it can be concluded that the candidate stimuli control the angular position of firing fields relative to the surroundings and are responsible for field stability. Informally, it could be said that the place cell system treats the environment as the same regardless of the angular position of the critical stimuli, 
with the result that fields rotate relative to the fixed laboratory frame when the stimuli are rotated.

As an aside, we note that control over the angular position of place cell discharge is not limited to visual stimuli. In some of the seminal experiments from O'Keefe's laboratory, it was demonstrated that auditory and somatosensory stimuli can support place cell firing (O'Keefe and Conway, 1978), and later work using similar stimuli, as well as olfactory cues, showed that they were effective in controlling the angular position of firing fields (O'Keefe and Speakman, 1987). Recent work shows that stimuli that activate the vestibular system also can be effective, although visual stimuli tend to be prepotent if they are in conflict with vestibular stimuli (Sharp et al., 1995).

In general, the stimuli used in previous work on angular control of firing fields have been of two kinds. In the experiments of O'Keefe and his colleagues, the stimuli are "distal" with respect to the portion of space accessible to the animal; distal means that the rat cannot make direct contact with the stimulus objects. The other kinds of stimuli have been flat objects (cardboard sheets) pasted to the walls of circular or rectangular apparatuses (Muller and Kubie, 1987). Such stimuli may be referred to as markers. Because the white cue card is on the cylinder wall, the rat can come into contact with it. Nevertheless, the card is not a "proximal" stimulus in the usual sense of the term. The card does not simply trigger place cells the fields of which are in its immediate vicinity. Instead, the card controls the angular position of firing fields regardless of whether the fields are adjacent or far from the card. Thus, the rather common distinction between distal and proximal cues cannot be used properly to describe the nature of the cue card.

Despite the differences between distal and marker stimuli, it is important to note that they have in common the property that they can be viewed only from a restricted range of viewpoints. It is not implausible, however, that rats might be able to navigate by using objects inside the arena as cues, in which case the cues could be seen from all viewpoints. It is known, for example, that rodents can locate a virtual point relative to two tall, narrow cylinders in an otherwise featureless environment (Collett et al., 1986; Gothard et al., 1996). It is also plausible that stimuli of this kind, which we will refer to as landmarks, also might be able to control the angular position of firing fields.

The present study was undertaken in light of these considerations. Specifically, we asked whether the angular position of place cell firing fields would be controlled by large threedimensional objects placed near the center of the same cylinder used in the cue card experiments. The restriction of potential polarizing information to these landmarks is achieved by placing the cylinder in a cue-controlled environment. The basic experiment is to compare the angular location of firing fields under two conditions. In the first, a set of objects near the apparatus center is in a "standard" position; in the second, the objects are rotated as a rigid set around the center of the cylinder. The surprising outcome of this experiment is that firing field angle is not coupled to the angular position of the landmarks. If, however, the very same objects are repositioned in certain ways in the cylinder, they exert nearly ideal stimulus control over field angle, proving that the landmarks are detectable by the rat. The fact that the objects do not control field angle when centrally located has interesting implications concerning the computational capacities of the rat navigational system.

\section{MATERIALS AND METHODS}

The methods used here are the same as those used by Muller et al. (1987). We first summarize the methods common to the two experiments reported in this paper. Methods specific to each experiment are described later.

Subjects. Long-Evans male rats (Janvier, St. Berthevin, France) weighing between 300-350 gm were used. They were housed one per cage in a room on a natural light-dark cycle (temperature, $20 \pm 2^{\circ} \mathrm{C}$ ). They had water ad libitum during all phases of the experiment. On receipt, the rats were handled daily for 2 weeks before presurgery training. So that positional firing rates everywhere in the cylinder could be estimated, the rats were food-deprived to $85 \%$ of ad libitum body weight and then trained in a "pellet-chasing" task for $10 \mathrm{~d}$. In this task, the rat had to retrieve $20 \mathrm{mg}$ food pellets scattered into the cylinder. The pellets were delivered via an automatic food dispenser located $2 \mathrm{~m}$ above the cylinder. The dispenser was equipped with five small tubes through which the pellet could drop onto the floor. Because the food pellets landed in unpredictable places, the rat learned to run almost constantly over the whole floor surface. After training was complete, the rat visited the entire floor area in just a few minutes and so covered the accessible area several times during a $16 \mathrm{~min}$ recording session. The objects that were used during the recording were in place during the presurgery training period. No attempt was made to "disorient" the rats during training (see Knierim et al., 1995).

Apparatus. The arena was a gray cylinder $50 \mathrm{~cm}$ high and $76 \mathrm{~cm}$ in diameter. The cylinder was isolated visually from the rest of the laboratory by a concentrically placed cylindrical curtain $250 \mathrm{~cm}$ in diameter and height. The floor of the cylinder was a piece of gray paper that was replaced between sessions. During both screening and recording sessions, four FM radios (one at each corner of the experimental room) tuned to the same frequency were switched on to mask possible uncontrolled auditory cues. The rats were introduced into the recording cylinder from one of four equally spaced positions around the circumference. The entry position for a given session was chosen from a list of random numbers. We found no effect of entry position on firing field position.

Three landmark objects were used, two for some rats and all three for others. The objects differed from each other in color, size, shape, and texture. For a given rat, their number, locations relative to each other, and their distances from the cylinder wall were fixed. The objects were a black wooden cone (height, $25 \mathrm{~cm}$; diameter, $11 \mathrm{~cm}$ ), a white plastic cylinder (height, $25 \mathrm{~cm}$; diameter, $10 \mathrm{~cm}$ ), and a bottle of French red wine (1993 vintage; height, $28 \mathrm{~cm}$; diameter, $9 \mathrm{~cm}$ ).

Two configurations of the objects were used. In the "two objects" configuration, the objects were the black cone and the white cylinder. In their "standard" arrangement, the point midway between the two objects was at the center of the cylinder. The two objects were $25 \mathrm{~cm}$ apart along the horizontal diameter of the cylinder as viewed from the overhead TV camera, with the cone to the left of the cylinder. In the "three objects" configuration, the third object (the wine bottle) was added to form an isosceles triangle, the height of which was $15.6 \mathrm{~cm}$ and the base of which was $25 \mathrm{~cm}$. The base of the triangle was along the horizontal diameter of the cylinder, with the cone to the left and the white cylinder on the right; each object was $12.5 \mathrm{~cm}$ from the cylinder center. For some rats a variant of the standard three objects configuration was used, with the objects rotated as a rigid set $45^{\circ}$ counterclockwise with respect to the more usual configuration.

Surgery. Surgery was done after training in the pellet-chasing task was complete. An injection of $0.3 \mathrm{ml}$ of atropine was given to prevent respiratory distress. Next, rats were anesthetized with pentobarbital (45 $\mathrm{mg} / \mathrm{kg}$ ) and placed in a Kopf stereotaxic apparatus. After a midline incision of the scalp, the skin and the muscles were retracted, and holes were drilled in the skull at appropriate locations. A movable array of ten $25 \mu \mathrm{m}$ electrode wires (Kubie, 1984) was implanted stereotaxically in the dorsal hippocampus at the following stereotaxic coordinates: $3.8 \mathrm{~mm}$ posterior and $3.0 \mathrm{~mm}$ lateral to bregma and $1.5 \mathrm{~mm}$ below the dura (Paxinos and Watson, 1986). Miniature screws were placed over the right olfactory bulb, the left frontal cortex, and the left cerebellar hemisphere. An additional screw with the head ground to a T-shape was lowered upside down into another hole in the left parietal bone and turned $90^{\circ}$ before being locked in place with a nut. For protection from the dental cement, sterile petroleum jelly was applied to the exposed brain surface and the guide tubing of the electrode array. Dental cement was applied over the jelly and around the guide tubing. The exposed skull was covered with dental resin cement. Then the screws and nut were embedded in 
dental cement, and the bottoms of the three drive-screw assemblies were cemented to the skull.

At the completion of the experiment, animals were killed with a lethal dose of pentobarbital and perfused intracardially with $0.9 \%$ saline, followed by $4 \%$ formalin. Just before death, positive current $(15 \mu \mathrm{A}$ for 30 sec) was passed through one of the microwires to deposit iron that could be visualized after reaction with potassium ferrocyanide (Prussian blue) The brains were removed and stored for $1 \mathrm{~d}$ in $3 \%$ ferrocyanide. Later, frozen coronal sections $40 \mu \mathrm{m}$ in thickness were taken. Every fifth section was stained with cresyl violet for verification of electrode placements.

Recording methods. Beginning 1 week after surgery, the activity from each microwire was screened daily while the rat chased pellets in the cylinder. The electrodes were lowered over a period of several weeks while searching for unit waveforms of sufficient amplitude to be isolated. Once a unit was isolated, it was recorded during several 16 min pelletchasing sessions. Such multiple sessions are possible because the same cell can be recorded reliably for days or even weeks (Muller et al., 1987). This makes it possible to compare the firing of an individual cell after the environment has been changed many times.

Screening and recording were done with a cable attached at one end to a commutator that allow the rat to turn freely. The other end of the cable was connected to an LED for tracking the rat's head position, a headstage with a field effect transistor amplifier (FET) for each wire, and, finally, a connector that mated with the electrode connector cemented to the rat's skull. The FETs were used to amplify signals before they were led to the commutator via the cable. The fixed side of the commutator was connected to a distribution panel. From the panel the desired signals were amplified 1000-fold with low-noise differential amplifiers and bandpassfiltered from $0.3-10 \mathrm{kHz}$. Then the signals were sent to two time-andamplitude window discriminators (Model DIS-1, Bak electronics) arranged in series for unit isolation. Accepted spikes were converted to digital pulses that were counted for $20 \mathrm{msec}$ intervals. At the end of each such interval (the end of a TV frame), the spike count for one or more cells was sent as a 4-bit binary number to a computer.

In addition to spike data, the rat's head position was tracked by locating a red LED, which was positioned on the midline $\sim 1 \mathrm{~cm}$ above the head and somewhat forward of the rat's eyes. The LED was tracked with a TV-based digital spot follower. The $x$ and $y$ positions of the LED were stored at 8 bits each so that the LED was detected in a grid of $256 \times 256$ square regions (pixels) $6.25 \mathrm{~mm}$ on a side. For calculating positional dwell time, distributions, and positional firing patterns, the resolution was reduced by 2 bits in each dimension, yielding a $64 \times 64$ grid of pixels 25 $\mathrm{mm}$ on a side. The $x$ and $y$ coordinates at the end of each frame were stored in parallel with the number of spikes counted during the $20 \mathrm{msec}$ frame. At $50 \mathrm{~Hz}$, a total of 48,000 sequential samples of position and associated spike count were accumulated in a 16 min recording session.

Testing protocol. The 10 electrodes in each rat were checked several times a day while the rat was in the cylinder. If no cell could be isolated, the electrode bundle was advanced $25-50 \mu \mathrm{m}$. Cells selected for recording were well discriminated complex spike cells that showed clear location-specific firing. Once a unit was well isolated, several recording sessions were run in a row. Before each session the waveform and firing pattern were inspected to check for constancy. Between sessions, rats were returned to their home cages, the objects were removed from the apparatus, and the floor paper in the cylinder was replaced. Next, the objects were placed at appropriate locations in the cylinder. To do this, a small light was mounted on the top of each object. The $x$ and $y$ coordinates of the light were read from the computer. When it was necessary to rotate the object set (see below), the new coordinates for each object were calculated, and the light was used to put each into its desired position. This procedure ensured that the positions of the objects relative to each other and their distances from the cylinder wall were held constant so that the objects in principle could act as reliable spatial cues.

In both experiments recordings were made first with the objects in a "standard" position relative to the laboratory frame and next with the objects rotated as a rigid set around the center of the cylinder. Usually, two sessions with the objects in the standard position were made. The purpose of these standard sessions was to ensure that the position of the firing field of the cell was stable under constant conditions. If this was the case, several "rotation" sessions were done during which the set of objects was in a rotated position. Rotations of the object set were usually $90^{\circ}$, although $15^{\circ}$ rotations were done for a few cells in Experiment 1. Occasionally, the object set was returned to the initial standard conditions after rotations were finished.

In the event that the firing field of a cell was unstable between the initial two standard sessions, at most two additional standard sessions were conducted. In any event, no rotation sessions were done until a firing field was stable for two standard sessions in a row.

Data presentation and analyses. Data were analyzed off-line. To obtain a positional firing rate distribution, the total time the red light was detected in each pixel (dwell time) and the total number of spikes in each pixel were accumulated for the session duration. The rate in each pixel was the number of spikes divided by the dwell time. Color-coded firing rate maps were used to visualize positional firing rate distributions. In such maps yellow pixels represent locations in which the firing rate was exactly $0.0 \mathrm{~Hz}$ for the whole session. The highest firing rate category is coded as purple. Intermediate firing rates are shown as orange, red, green, and blue pixels from low to high. Pixels that were never visited during a session are encoded white.

Because the in-field firing rates of place cells can vary over a large range, the values used as boundaries between color categories were autoscaled for the map of the first session recorded for a given cell. To permit comparisons among positional firing distributions across several sessions for a cell, we used rate categories for subsequent sessions that were the same as for the first session.

Inspection of the positional firing patterns across successive sessions was used to classify each cell into one of the following two categories.

(1) Cells for which the angular position of the firing field could be predicted from the angular position of the stimulus ensemble. To be included in this category, the shape, size, intensity, and angular position of the firing field had to be stable across successive standard sessions. In addition, the firing field had to rotate appropriately when the stimulus ensemble (either the objects alone or the objects plus the cue card, depending on the current condition) was rotated. A firing field was said to rotate appropriately when its angular position moved through an angle that was within $6^{\circ}$ of the angle through which the stimulus ensemble was rotated. Because the angular resolution was $6^{\circ}$, this means that the field was at most one angular bin away from that bin expected for perfect control by the stimulus ensemble.

(2) Cells for which the angular position of the firing field was unrelated to the position of the stimulus ensemble. The lack of relationship could occur either during successive standard sessions or during stimulus rotation sessions. For some cells the angular position of the field changed between pairs of standard sessions, and the cell was judged immediately to be uncontrolled. More precisely, if the angular position of the field was different in the first two standard sessions, a third standard session was done. If the angular position of the field in the third standard session was different from that in the second standard session, the cell was judged uncontrolled and no additional recordings were made. On the other hand, if the angular position was stable between standard sessions 1 and 2 or 2 and 3 , rotation sessions were done to assess control by the stimulus ensemble; judgments were made according to the criteria stated above.

To estimate numerically the firing field rotation between session pairs, we calculated a pixel-by-pixel cross-correlation as the positional firing pattern for the second session was rotated in $6^{\circ}$ steps relative to the positional firing pattern for the first session. That is, the pixel-by-pixel cross-correlation was calculated 60 times at rotations of $0,6,12 \ldots$ degrees. The rotation associated with the highest correlation $\left(R_{\mathrm{MAX}}\right)$ was taken as the rotation of the firing field between the two sessions. Counterclockwise rotations were taken as positive and clockwise rotations as negative. The difference between the rotation expected if the angular field position were controlled perfectly by the stimulus ensemble and the observed rotation was the estimate of rotation error for a pair of sessions. If the field rotated less than expected, the error was taken as negative; if the field rotated more than expected, the error was taken as positive. Because $180^{\circ}$ cue rotations were never done, the ambiguity of how to define the sign of the error presented no problem.

\section{EXPERIMENT 1}

In the first experiment we sought to determine whether two or three objects placed in a fixed configuration relative to each other and near the center of the cylinder could exert reliable stimulus control over the angular positions of firing fields. For three rats recordings were made with two objects near the center of the apparatus ("two objects" condition), whereas for four others recordings were made with three objects arranged around the center of the apparatus ("three objects" condition). The two 
Table 1. Comparison of spatial firing characteristics in objects-only and objects + card conditions

\begin{tabular}{|c|c|c|c|}
\hline & Spatial coherence & Information content & Place field size \\
\hline Two objects & $0.61 \pm 0.04$ & $1.91 \pm 0.12$ & $83 \pm 7.6$ \\
\hline Three objects & $0.69 \pm 0.03$ & $2.09 \pm 0.15$ & $72 \pm 6.9$ \\
\hline Objects + card & $0.57 \pm 0.04$ & $2.38 \pm 0.18$ & $73 \pm 10.0$ \\
\hline
\end{tabular}

Values are given as means \pm SE. The total size of the cylinder was $\sim 725$ pixels.

objects and three objects conditions are referred to together as the "objects-only" condition.

Because it was found the objects alone in fact did not control the angular position of firing fields reliably, additional sessions were done for some rats after adding a white cue card to the stimulus ensemble. The white card was chosen because it was known from previous work to exert stimulus control over angular position (Muller and Kubie, 1987). The rats initially tested with two objects later were tested with both the objects and a cue card attached to the cylinder wall ("objects plus card" condition). The cue card was a white cardboard sheet that covered $100^{\circ}$ of wall arc. Its angular position was fixed relative to the object set. Because the card was added $\sim 1$ month after the beginning of objects-only recordings, 1 week was allowed for familiarization before subsequent recordings.

\section{Results}

\section{General observations}

Histological analysis revealed that electrodes were placed in the dorsal hippocampus of all animals. Recordings were made from 83 hippocampal complex spike cells that were judged to be robust place cells by inspection of color-coded rate maps. No attempts were made to record from complex spike cells that fired at very low rates (silent cells) nor from cells that fired at an appreciable rate everywhere in the apparatus. No systematic attempt was made to determine the fraction of cells with discriminable waveforms that were place cells. Of the 83 cells, 12 were excluded from analysis either because they were lost before enough data were gathered to categorize the cell ( 8 units) or because the waveform changed so much that we could not be confident it was the same unit; changes of this type usually occurred while we were connecting or disconnecting the rat from the recording cable.

The remaining 71 cells were held for enough recording sessions to allow a decision as to whether the stimulus ensemble did or did not have control over the angular position of firing fields. Some general results should be mentioned first. For one thing, the positional firing patterns of cells in standard sessions with two objects $(n=25)$ appeared to be the same as for cells in standard sessions with three objects $(n=27)$. Specifically, the mean values of spatial coherence (Muller and Kubie, 1989), spike information content (Jung and McNaughton, 1993), and place field size (Muller et al., 1987) were indistinguishable for two and three object standard sessions (Table 1). Inspection of firing rate maps suggested no differences between two object and three object firing patterns that might have been undetected by the numerical measures. The similarity of positional firing patterns suggests that the number of objects near the cylinder center does not affect the positional firing characteristics of place cells. A second general finding is that there were no differences seen between cells recorded in standard objects plus card sessions $(n=19)$ and standard objects-only sessions. Accordingly, it is appropriate to compare stimulus control in the objects plus card and objects-only conditions.
Table 2. Cumulative number of cells departing from object control as a function of session sequence in each condition

\begin{tabular}{lcccc} 
& $\begin{array}{l}\text { Standard to } \\
\text { standard }\end{array}$ & $\begin{array}{l}\text { Standard to } \\
\text { rotate }\end{array}$ & $\begin{array}{l}\text { Rotate to } \\
\text { standard }\end{array}$ & Total $(\%)$ \\
\hline Objects only & $16 / 52$ & $44 / 52$ & $50 / 52$ & 96.1 \\
Objects + card & $2 / 19$ & $2 / 19$ & $2 / 19$ & 10.5 \\
Clustered objects & $0 / 19$ & $0 / 19$ & $0 / 19$ & 0.0 \\
Objects at periphery & $0 / 16$ & $0 / 16$ & $0 / 16$ & 0.0 \\
\hline
\end{tabular}

\section{Recordings made in the objects-only condition}

Of the 52 cells recorded in the objects-only condition, 50 had firing fields for which the angular position could not be predicted consistently from the angular position of the object set. Such firing fields were either unstable during standard sessions, were fixed relative to the laboratory frame after object rotation, or had unpredictable angular positions during rotation sessions. The "session sequence" (initial standard sessions, rotation session, final standard session) for which the observed angular field position first departed from the position predicted from object control was determined for each cell by visual inspection of firing rate maps. The first departure occurred during the standard sequence for $16 / 52$ units, during the rotation sequence for $28 / 50$ units, and during the final standard session for $6 / 52$ cells. Cumulative values for the number of cells departing from object control are shown in Table 2.

For only two cells (4\%) recorded in the objects-only conditions were the angular firing field positions controlled by the object set. Although the two cells were obtained from two different rats in the three objects condition, a $\chi^{2}$ test did not suggest there was any systematic difference between the two objects-only conditions $\left(\chi^{2}\right.$ $=1.80 ; \mathrm{df}=1 ; p=0.18, \mathrm{NS})$. This suggests that the number of objects did not affect their ability to control the angular position of firing fields. Accordingly, the data from the two objects and three objects conditions were pooled. Similarly, the rarity of stimulus control precludes the possibility that differences would exist between CA1 $(n=27)$ and CA3 $(n=25)$ place cells so that all place cells are treated the same.

The general ineffectiveness of the object set in controlling the angular position of firing fields is summarized in the scatterplot of Figure $1 A$. The "expected angular position" of a firing field was derived by adding the amount of object rotation to the observed angular position of the firing field for a baseline session. The expected angular position is plotted on the $x$-axis. The $y$-axis is the observed angular position of the fields for the next session. If the angular position of the objects controlled the angular position of the fields, the points would all lie along the $45^{\circ}$ line. From Figure $1 A$ it is evident that there was little control. The lack of points along the $45^{\circ}$ line occurs because only $2 / 52$ cells showed stability across the entire preliminary four session (standard, standard, rotation, standard) sequence. For each of the other 50 cells (Table 2), control by the objects failed earlier in the preliminary sequence. At this point the sequence was halted. The session preceding the failure was taken as the baseline session, and the session in which the failure occurred was taken as the next session. The circular correlation coefficient (Batschelet, 1981) for the 52 points was 0.15 , showing that the position of the object set does not predict the firing field angular position.

The nature of the lack of stimulus control by the objects is illustrated in Figures 2 and 3. The most dramatic kind of failure of the objects to control the angular position of fields occurs under 
(A) objects-only

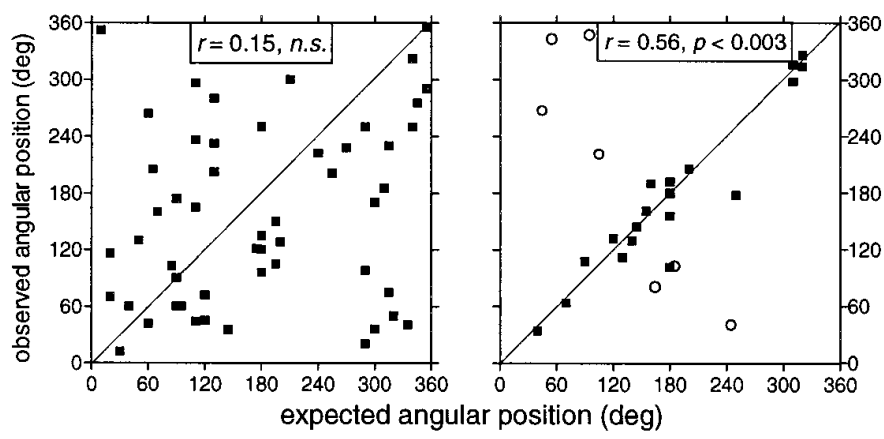

Figure 1. Scatterplot of expected versus observed angular positions of firing fields. Expected angular position of firing fields is shown on the $x$-axis; observed angular positions are shown on the $y$-axis. Correlation coefficients $(r)$ for circular data were calculated according to Batschelet (1981). A, Objects-only condition. B, Objects plus card condition (black squares, objects and card-coefficient of correlation calculated on these data; open circles, objects only).

constant conditions. For some cells the field is seen to rotate to seemingly arbitrary angular positions between pairs of standard sessions. Two examples of unstable fields under constant stimulus conditions are shown in Figure 2. In Figure $2 A 1$ a large, intense firing field appears at approximately 5:30 o'clock. In the second standard session (Fig. $2 \mathrm{A2}$ ) the field has shifted by $180^{\circ}$ to approximately 1:00 and is divided into two parts by the wine bottle. In the third standard session (Fig. 2A3) the field has jumped by $-120^{\circ}$ to approximately 8:00. For this cell there seems to be no tendency of the field to stay in register with the set of three objects nor even with uncontrolled cues fixed in the laboratory frame ("static background cues"; O'Keefe and Speakman, 1987). The second cell in Figure $2 B$, recorded from a different rat, showed similar lack of stability under fixed stimulus conditions.

Even if the firing field of a cell were stable during a series of standard sessions, rotation experiments usually revealed that the objects did not exert reliable stimulus control over the angular position of firing fields. The lack of stimulus control could be manifested in a variety of ways, three of which are illustrated in Figure 3. The field of the cell in row A of Figure 3 was unstable between the first two standard sessions, where it rotated by $-66^{\circ}$. In contrast, the field rotated only $-6^{\circ}$ between the second and third sessions. The impression is that the angular field position stabilized by the time of the third session. In fact, this cell satisfied the first stability criterion, namely that the firing field had to be in a fixed position for at least one consecutive pair of standard sessions. A session done after stimulus rotation revealed, however, that the objects had no control over the angular field position; when the cues were rotated $+90^{\circ}$, the field rotated $+162^{\circ}$, for an error of $+72^{\circ}$. Furthermore, when the cues were rotated back to standard position, the field rotated back to near its initial position and not to where it was just before the rotation.

The second cell illustrated in Figure 3 (row $B$ ) had a stable field for the first two standard sessions and was, therefore, immediately a candidate for rotational testing of stimulus control. When the objects were rotated $+90^{\circ}$, the field rotated $174^{\circ}$ (rotation error $=$ $+84^{\circ}$ ) to a completely unexpected angular position. When the objects were returned to the standard position, the field rotated only $-42^{\circ}$ (rotation error $=-48^{\circ}$ ). Yet another pattern was seen for the cell in row $\mathrm{C}$ of Figure 3. The field was again stable during the first two standard sessions. A $+90^{\circ}$ object rotation resulted in only a $48^{\circ}$ field rotation (rotation error $=-42^{\circ}$ ). For this unit, returning the objects to their standard position was associated with field rotation back to its initial position.

A total of 101 rotation sessions was conducted. The firing field rotated an unexpected amount in 43 rotation sessions and stayed fixed relative to the laboratory frame (and not the object set) in 39 other sessions. In only 18 rotation sessions did the field rotation match the rotation of the object set. Ten of the 18 sessions are likely happenstance, in which the field rotated randomly to a position close enough to that expected from rotational control by the objects; we believe this to be the case because these sessions were recorded from cells for which the firing was not controlled consistently by the object set.

The remaining sessions in which there was apparent control of firing field position by the object set were done for a single cell and for a pair of simultaneously recorded cells. In each case there was never a departure of the observed angular location of the firing field from that expected, given ideal control by the object set, so that both stability criteria were satisfied. For the individual cell (data not shown), the rotation error after a $+90^{\circ}$ rotation was $0^{\circ}$, and after a subsequent $-90^{\circ}$ rotation was $-6^{\circ}$. The paucity of rotation sessions for this cell and the fact that the second rotation returned the cue set to the standard position leaves open the possibility that additional testing for this cell would have revealed instability.

In contrast to the individual cell, a total of six rotation sessions was done for a pair of cells simultaneously recorded from a single electrode (Fig. 4). The waveforms of these cells were too similar to separate with the window discriminators reliably, so the firing of both is shown together in single rate maps. Because the circular field of one cell was almost centered in the cylinder, it was difficult to assess the effects of rotations on its angular position. The other cell, however, had a crescent-shaped field, the angular position of which could be visualized easily and measured. The effects of a sequence of sessions with $15^{\circ}$ and $90^{\circ}$ rotations of the object set are shown in Figure 4. By inspection of the maps, the firing field rotation was very similar to the rotation of the objects. Numerically, the mean rotation error was $-2.5^{\circ}$. The small average rotation error shows that the angular position of the crescentshaped field was controlled precisely by the angular position of the object set. In Discussion, we consider the significance of this departure from the rule of lack of control by the stimulus objects.

\section{Recordings made in the objects plus card condition}

Because the objects by themselves did not control the angular position of firing fields reliably, an additional set of recordings was done after we attached a white cue card to the gray cylinder wall. This modification allowed us to ask two questions. First, could the card, in conjunction with the objects, consistently control the angular position of field fields? It was known from previous work that the card by itself can exhibit virtually ideal control over firing fields so that an inability in the current circumstances would imply that the objects were not only ineffective but also deleterious to control. The second question assumes that consistent control is established in the presence of the objects plus card. If so, would withdrawing the card return matters to the original state in which the objects by themselves were ineffective? Alternatively, could some association between the card and the objects be formed that would "transfer" control to the objects?

So that we could answer these questions, the three rats tested in the two objects condition received a week of additional training in the pellet-chasing task, with the card present in a fixed position 

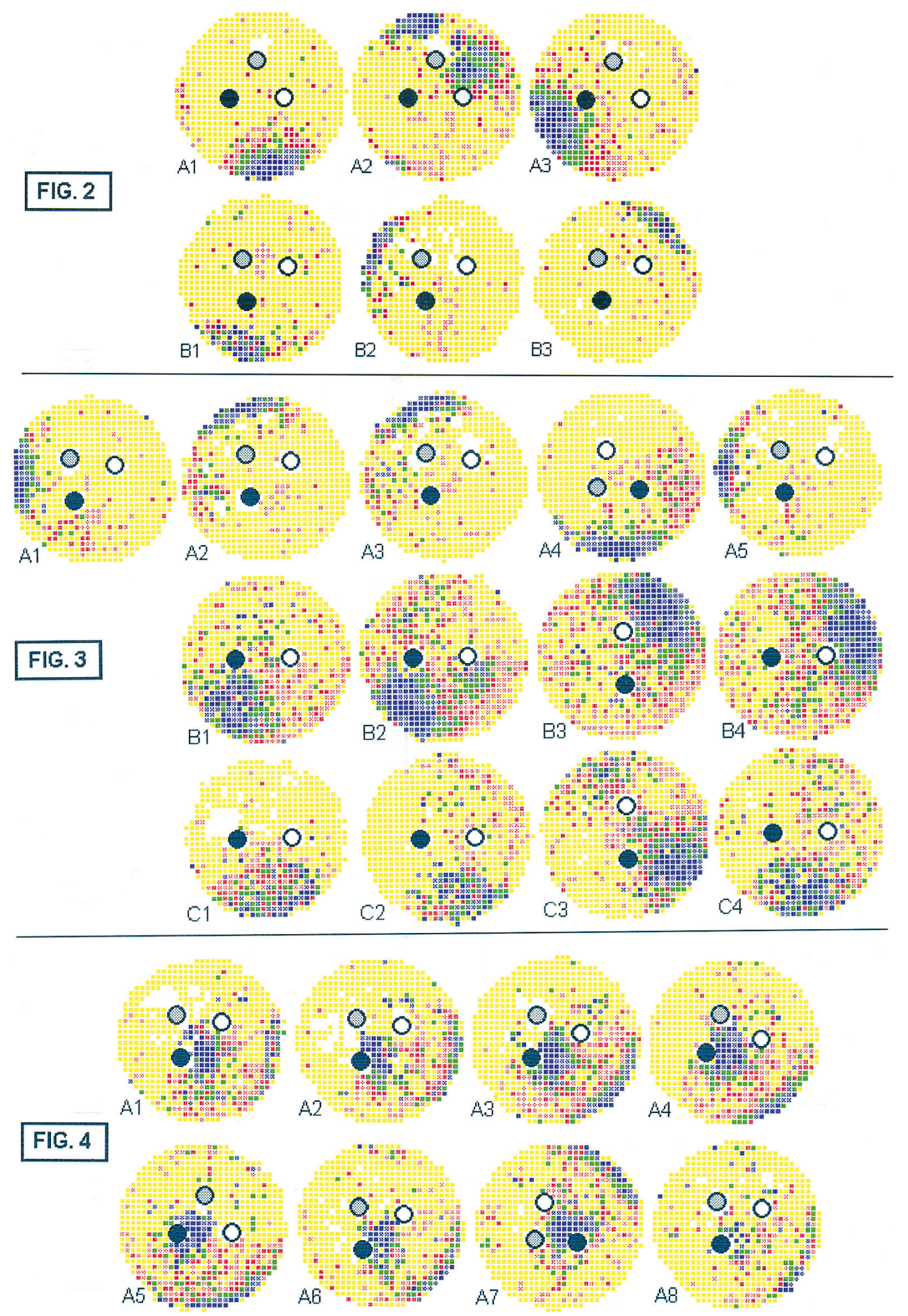

Figure 2. Top. Firing rate maps of two cells recorded during three consecutive standard sessions in the objects-only condition. In this and subsequent figures, the black cone is shown as a black circle, the white cylinder as a white circle, and the wine bottle as a gray circle. In the two cells the position of firing fields changed across standard sessions under constant conditions. Color codes for a given cell are based on the firing activity of the cell during the first recording session. Median firing rates for colors: $A$, yellow, 0.0; orange, 0.5 ; red, 1.1; green, 2.5; blue, 8.7; purple, 16.4 action potentials per second $(\mathrm{AP} / \mathrm{sec}) . B, 0.0 ; 0.5 ; 1.0 ; 2.2 ; 6.2 ; 15.9 \mathrm{AP} / \mathrm{sec}$. 
relative to the objects. Because the card was introduced after recordings had been done with only the objects present, the electrode array already had been advanced. The consequence is that all cells recorded in the objects plus card condition were from CA3, whereas recordings made in the objects-only conditions were from CA1 and CA3.

Of the 19 cells recorded in the objects plus card condition, the angular field positions of 17 clearly were controlled by the stimulus ensemble. The fields of the other two cells remained fixed relative to the laboratory frame and presumably were controlled by static background cues. The response of firing fields to stimulus ensemble rotations is shown with filled squares in the scatterplot of Figure $1 B$, in which the points for the two uncontrolled units are evident.

The rate maps for a typical cell in the objects plus card condition are shown in maps in the top row of Figure 5. For the first two standard sessions the angular position of the firing field was stable; the rotation error was $-6^{\circ}$. When the objects plus card were rotated $+90^{\circ}$, the field rotated $84^{\circ}$, for a rotation error of $-6^{\circ}$. Returning the stimulus ensemble to its initial position caused the field to rotate back (rotation error $=-6^{\circ}$ ). We conclude that the objects did not disrupt the ability of the card to control the angular position of firing fields.

Once it was established that the objects plus card exerted effective stimulus control, it was important to test whether the objects alone now would exert control. Of the 17 cells that were stable in the objects plus card condition, seven were held long enough to test the effects of removing the card. In no case was the angular field position of any of these cells controlled by angular position of the objects. The ineffectiveness of the objects in controlling firing field position in this repetition of the objectsonly condition is shown with open circles in the scatterplot of Figure $1 B$.

Examples of the lack of control are shown in the maps in the bottom row of Figure 5. Simply removing the card and leaving the objects alone resulted in a stable firing pattern (rotation error $=$ $+6^{\circ}$; compare maps $A 4$ and $A 5$ in Fig. 5). In the next session, however, a $+90^{\circ}$ rotation of the objects caused a $-60^{\circ}$ rotation of the firing field (rotation error $=-150^{\circ}$ ). When the objects were returned to their initial position in the next session with a $-90^{\circ}$ rotation of the objects, the field rotated $-150^{\circ}$ (rotation error $=$ $+60^{\circ}$ ). When the card was replaced in its usual position relative to the objects, the angular location of the firing field snapped back to its original location (see map $A 8$, Fig. 5). The same pattern of results was seen for the other six cells tested this way. It therefore

\footnotetext{
Figure 3. Middle. Firing rate maps of three cells recorded during consecutive sessions in the objects-only condition. The object set was left in standard position until the field was stable and then was rotated $90^{\circ}$ (maps $A 4, B 3, C 3$ ). None of the three cells had a firing field the position of which was controlled consistently by the position of the object set. Median firing rates for colors (order as in Fig. 2): $A, 0.0 ; 0.7 ; 1.3 ; 2.1 ; 3.2 ; 6.1 \mathrm{AP} / \mathrm{sec} . B$, $0.0 ; 0.8 ; 1.8 ; 3.8 ; 7.8 ; 14.2 \mathrm{AP} / \mathrm{sec} . C, 0.0 ; 0.6 ; 1.3 ; 2.1 ; 3.6 ; 5.6 \mathrm{AP} / \mathrm{sec}$.

Figure 4. Bottom. Firing rate maps of a cell recorded during eight consecutive sessions in the objects-only condition. After two standard sessions, the object set was rotated in $-15^{\circ}$ steps (sessions $3-5$ ) and then returned to its original position (session 6 ). Session 7 was a $+90^{\circ}$ rotation session and session 8 another standard session. Two cells were recorded simultaneously on the same electrode, but only the cell with a crescentshaped (peripheral) field can clearly demonstrate control by the object set. Median firing rates for colors (order as in Fig. 2 ): $0.0 ; 1.1 ; 2.3 ; 4.4 ; 7.1 ; 14.3$ $\mathrm{AP} / \mathrm{sec}$.
}

seems clear that no effective association is generated between the card and the objects such that stimulus control is "transferred" to the objects. For all seven cells tested, stimulus control returned when the cue card was put back in the cylinder.

\section{The effect of object rotation on the rat's behavior}

In previous work it was found that certain changes in the relationships among a set of objects would induce the rat to reexplore the objects (Poucet et al., 1986; Thinus-Blanc et al., 1987). Accordingly, we were interested in whether rotations of the object sets used in the present experiment could cause reexploration, although the object sets did not control the angular positions of firing fields. Because the behavioral effect, if any, is likely to be greatest the first time the objects are rotated, comparisons were made between the first rotation session and the immediately preceding standard session; the preceding standard session is referred to as the baseline session.

Reexploration was measured by accumulating the total time the rat spent in the vicinity of the objects during the first rotation session and comparing it with the baseline session. The area for accumulating time was an approximately circular region around each object. A pixel was included in its entirety if any part of it were in the annulus between the object and the outside of the circular region. The width of the annulus was set to $1,2,3,4$, and 5 pixel edge lengths to help eliminate the possibility that the results would depend critically on some accidental relationship between the object location and the pixel grid. ANOVA for each annulus size revealed no tendency whatsoever for there to be more time spent near the objects in the rotated session than in the preceding standard session (analyses not shown).

\section{EXPERIMENT 2}

Experiment 1 revealed that three-dimensional object sets placed near the center of the cylinder failed to control the angular position of firing fields, even after being paired with a white cue card that exerted strong control. There are several reasons that the objects might have been ineffective, and two rearrangements of the objects were made to help to decide among the possibilities. Note that rearranging the objects does not alter the fact that the objects present impediments to the animal's motions within the environment.

The first rearrangement may be referred to as the "clustered objects condition." In this circumstance the objects from the three objects condition were placed along a straight line such that the middle object (the wine bottle) touched both the "top" object (the black cone) and the "bottom" object (the white cylinder). Top and bottom refer to the standard position for the clustered objects arrangement. Specifically, the objects were aligned along a vertical chord of the cylinder, the midpoint of which was $23 \mathrm{~cm}$ from the center and such that the middle object was centered on the midpoint of the chord (see Fig. 7). Six naive rats were trained to chase pellets in the presence of the clustered objects.

The clustered objects arrangement was used to test the hypothesis that the objects simply could not affect the activity of place cells. The rationale was to set the objects so as to mimic the large size and eccentric placement of the cue card. If control over angular firing position could be achieved with such an arrangement, it would be clear that the objects were not effectively invisible to the place cell system.

The second rearrangement will be referred to as the "objectsat-periphery" condition. Here, the relationships among the three objects were identical to those in the three objects condition, 

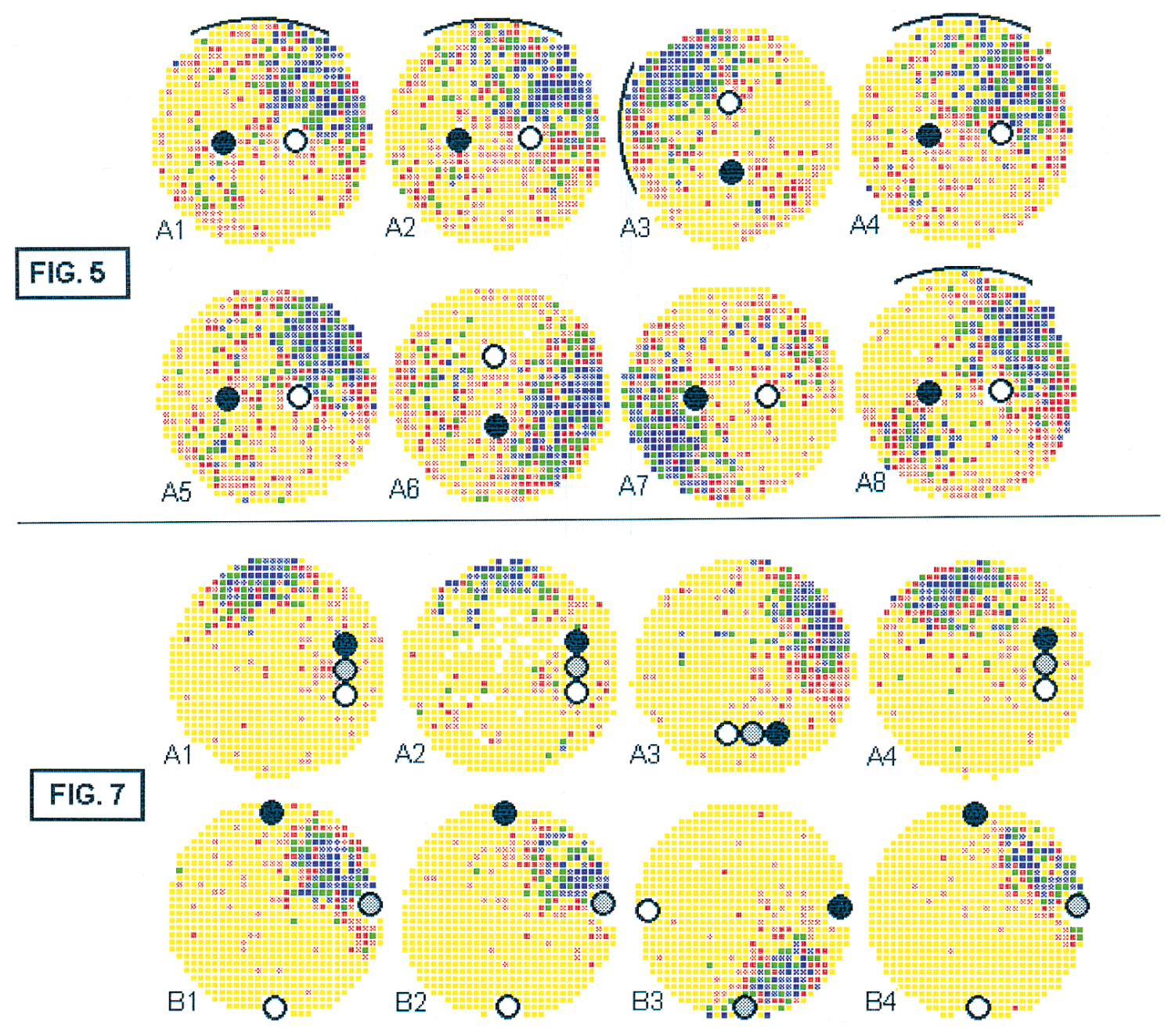

Figure 5. Top. Firing rate maps of a cell recorded in the objects plus card condition. In the presence of the white cue card, the position of the firing field was predicted from the position of the cue ensemble (sessions 1-4). Once the card was withdrawn, the position of the firing field became unpredictable (sessions 5-7). Returning the card (session 8) permitted restoration of the original firing field. Median firing rates for colors (order as in Fig. 2): 0.0; 0.6; $1.3 ; 2.6 ; 4.1 ; 8.3 \mathrm{AP} / \mathrm{sec}$.

Figure 7. Bottom. Firing rate maps of cells recorded during four consecutive sessions in the two conditions of Experiment 2. In both conditions the firing field was stable during standard sessions and was rotated appropriately after rotation of the object set (sessions $3-4$ ). $A$, Clustered objects condition. Median firing rates for colors (order as in Fig. 2): $0.0 ; 0.5 ; 1.3 ; 2.9 ; 5.4 ; 7.9$ AP/sec. B, Objects-at-periphery condition. Median firing rates for colors (order as in Fig. 2): $0.0 ; 0.6 ; 1.5 ; 2.5 ; 4.6 ; 8.7 \mathrm{AP} / \mathrm{sec}$.

except that the distances among the objects were scaled up such that each was against the wall of the cylinder. Thus, the objects once again formed an isosceles triangle, in this case oriented with the cone at 12:00, the bottle at 3:00, and the cylinder at 6:00 (Fig. 7). Here, the objects were separated as in the three objects condition, but they were placed in such a way that the rat could not go behind an object. The idea was to simplify the situation by making it impossible for the rat to view a pair of objects from two different perspectives such that one object was to the left of the other in one perspective and to the right of the other in the second perspective. Three naive rats were trained to chase pellets in the objects-at-periphery condition.

\section{Results}

Nineteen cells were recorded in the clustered objects condition and 16 in the objects-at-periphery condition. The angular position of the firing field was controlled by the objects for every cell in these two conditions; i.e., firing fields were stable during standard sessions and rotated appropriately during rotation sessions. Scatterplots showing the observed angular firing position against the position predicted by ideal control are shown for the clustered objects and objects-at-periphery conditions in Figure $6 A$ and $B$, respectively.

The top row of maps in Figure 7 shows the typical pattern of results observed in the clustered objects condition. The positional firing pattern was stable in the first two standard sessions. When the stimulus ensemble was rotated $-90^{\circ}$, the field rotated $-84^{\circ}$ (rotation error $=-6^{\circ}$ ). Returning the stimulus ensemble to the standard position caused the field to rotate back (rotation error $=$ $+6^{\circ}$.

The bottom row in Figure 7 shows the typical pattern of results observed in the objects-at-periphery condition. Once again, the positional firing pattern was stable in two standard sessions. Rotating the stimulus ensemble $-90^{\circ}$ caused the field to rotate $-90^{\circ}$ (rotation error $=0^{\circ}$ ). Returning the stimulus ensemble to 


\begin{tabular}{ll}
\hline Table 3. Average values of the correlation coefficients $\left(\boldsymbol{R}_{\text {MAX }}\right)$ \\
Condition & Mean $R_{\mathrm{MAx}} \pm \mathrm{SE}$ \\
\hline Objects only & $0.35 \pm 0.03$ \\
Objects + card & $0.31 \pm 0.04$ \\
Clustered objects & $0.34 \pm 0.05$ \\
Objects at periphery & $0.36 \pm 0.03$ \\
\hline
\end{tabular}

the standard position caused the field to rotate back (rotation error $=+6^{\circ}$ ).

\section{Effects of manipulations on firing field shapes}

Comparisons of the scatterplots in Figures 1 and 6 make it evident that stimulus control is absent only in the objects-only conditions of Experiment 1. The lack of stimulus control might come about in one of two ways. In the first, all characteristics of firing fields except angular position would be preserved. If this were true, firing fields would be recognizably the same regardless of how random angular position might seem to be in the objects-only condition. Inspection of the example maps in Figures 2 and 3 and the relevant part of Figure 5 strongly suggests that this is the case. The other possibility is that "remappings" were caused by rotations in the objects-only conditions. In a remapping (Quirk et al., 1990; Bostock et al., 1991) the firing field of a cell in one condition is unrelated to the field in a second condition by any simple transform, such as rotation. In addition, the cell may even virtually cease firing in the second condition.

It is, therefore, possible that the inconstancy of the angular position of firing fields in the objects-only conditions was associated with more sweeping changes in positional firing patterns than unpredictable shifts of the angular position. To test this possibility, we compared the average value of $R_{\mathrm{MAX}}$ for session pairs for each experimental condition. The idea is that $R_{\mathrm{MAX}}$ (see Data Presentation and Analyses) reflects the similarity between the positional firing patterns of session pairs. If the poor control of angular field position in the objects-only is associated with more extensive changes in positional firing pattern than in the other conditions, $R_{\mathrm{MAX}}$ is expected to be smaller.

The pairs used for analyzing $R_{\mathrm{MAx}}$ were the same as those used to summarize stimulus control in Figures 1 and 6; the exception is that the objects-only sessions that followed the objects plus card condition were not included. The results are summarized in Table 3 , from which it is evident that there were no differences in $R_{\mathrm{MAX}}$ across the conditions. We conclude that the lack of stimulus control in the objects-only conditions is not associated with major changes in the positional firing patterns. A corollary of this result is that the firing field location within objects-only sessions did not drift, as might be expected if the angular coordinate of field position depended on dead reckoning (Knierim et al., 1995); if such drift occurred, the field would be more diffuse and $R_{\text {MAX }}$ would be lower.

\section{DISCUSSION}

\section{General considerations}

In previous work it was shown that the angular positions of place cell firing fields can be controlled by stimuli that are inaccessible to a rat running on a radial arm maze (O'Keefe and Speakman, 1987) or by a single cue card on the wall of a cylindrical recording arena in which the rat retrieves randomly scattered food pellets (Muller and Kubie, 1987).

The purpose of this study was to test whether similar control
(A) clustered-objects

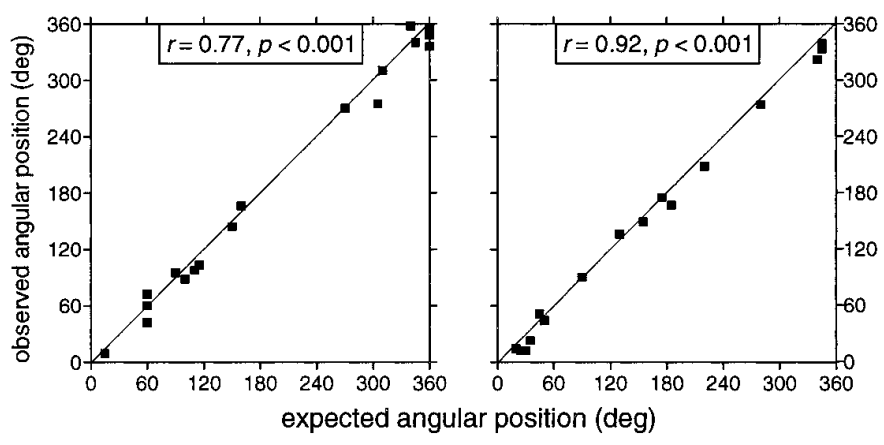

Figure 6. Scatterplot of expected versus observed angular positions of firing fields (see Fig. 1 for explanations). $A$, Clustered objects condition. $B$, Objects-at-periphery condition.

over firing fields could be exerted by three-dimensional objects placed directly in the cylinder. For almost all cells, no such control was exerted by ensembles of two or three objects set near the middle of the cylinder (objects-only conditions). Thus, the objects themselves, the particular arrangement of the objects in space, or both the objects and their arrangement make them less useful for anchoring the positional firing patterns of place cells.

It is important to realize, however, that the ineffectiveness of the objects-only arrangements is not absolute. Specifically, the angular position for the firing fields of 2 of 52 cells was controlled by the objects. Moreover, for one of these cells precise control was exerted over enough sessions to convince us that the objects near the center had, in fact, gained stimulus control. On the basis of this individual case we believe, therefore, that inability of the objects to control the angular firing position is not absolute. Instead, it is our argument that the rarity of such control reflects the complexity of the computations necessary to generate a representation relative to a set of objects that can be viewed from all directions. We will return to this theoretical issue after considering conclusions that can be drawn from other stimulus arrangements.

The finding of accurate stimulus control in the objects plus card condition has two related implications. First, it shows that the circumstances used in this study are similar enough to earlier work (Muller and Kubie, 1987) that virtually ideal control over the angular position of firing fields is possible. Second, it means that the three-dimensional objects are merely insufficient for reliable stimulus control but do not prevent such control. The fact that control was once again absent after the cue card was removed implies that control cannot be conferred on the objects even if they are kept for several sessions in a constant angular association with firing fields.

An additional finding from the objects-only condition is that firing fields tended either to rotate through an angle that had no clear relationship to the angular position of the object set or to stay in a fixed position relative to the laboratory frame (i.e., relative to a static background cue). In the first case, one imagines that the part of the navigational system responsible for setting the angular coordinate "selected" some unknown feature of the environment as a stable anchor; this occurred during $\sim 62 \%$ of the rotation sessions in the objects-only conditions. In the second case, the anchor is presumably a component of the stimulus ensemble provided by the fixed laboratory frame; this occurred in the remaining $38 \%$ of the objects-only rotation sessions. The 
inference is that there is a hierarchy of stimulus types used as anchors for the angular position of firing fields. The most powerful stimuli are easily discriminable ones on the cylinder wall. Next are static background cues from the laboratory frame. Finally, the object set is used rarely, despite its seeming salience.

Two additional experiments revealed that it was not the nature of the objects per se but, rather, their positional arrangement that was responsible for the lack of stimulus control in the objects-only conditions. Arranging the objects in a row to mimic the stimulus properties of the cue card (clustered objects condition) resulted in virtually ideal control over the angular positions of firing fields. This is sufficient to demonstrate that the objects are not simply "invisible" to the aspect of the navigational system revealed by place cells.

A more important demonstration of the crucial nature of the object arrangement is provided by the objects-at-periphery experiment in which the objects are separated once again to form a triangle similar to that in the three objects condition. Here, however, the triangle is dilated such that each object is against the cylinder wall. Under these circumstances the objects exert virtually ideal control over the angular position of firing fields, suggesting that the lack of control in the three objects condition has to do with the size and central location of the object triangle. A future experiment of considerable interest would be to change the size of the triangular object arrangement to determine how far from the walls it would be necessary for the objects to be moved before stimulus control was lost. According to one line of speculation about the lack of control with the objects at the center, the loss of control would occur as soon as the rat could move between each object and the nearest point on the wall (see below). Another future experiment would be to change the object triangle from right isosceles to equilateral to test whether the individual objects can be recognized separately or whether it is the asymmetric layout in space that permits the navigational system to use the object group as an anchor for the angular coordinate. The same information could be gained by putting two objects against the wall on a diameter.

\section{Why is the stimulus control exerted by the objects so weak?}

Because it is clear that the objects themselves are detected by the rat, it is a critical question as to why they are not used to anchor the reference direction when they are near the center of the cylinder. The answer we propose is that the computations are too difficult for the rat brain, because the relationships between pairs of objects change in fundamental ways as the rat moves around in the cylinder. For example, because the rat can see each pair of objects from any angle, one object of the pair may be either to the left or right of the other, depending on the rat's current position. In addition, when the rat's position is along the line that connects a pair of objects, the more distant object is eclipsed by the nearer one; even the availability of the individual objects is variable. Also, there are difficulties when the rat goes between a pair of stimuli. For the two objects condition, the handedness of the two objects relative to the rat at a given position is reversed compared with what exists if the rat approaches the two objects from the other side. For the three objects condition, things are even more difficult; the relationships among the objects are very different if the rat is inside the triangle rather than outside.

None of the stated difficulties arise if it is impossible for the rat to move around stimuli that potentially can be used to anchor the reference direction. The distal stimuli used in O'Keefe's work
(O’Keefe and Conway, 1978; O'Keefe and Speakman, 1987) and the cue card used here and by Muller and Kubie (1987) share this crucial property, namely that the rat cannot "get behind" stimuli used to anchor the angular reference direction. We propose that the objects at the periphery are effective in controlling the angular position of firing fields because they then provide exactly the same sort of simple, stable navigational information as is provided by the cue card.

One obvious difficulty with the proffered explanation is the ability of the clustered objects to control the angular position of firing fields despite the fact that the rat can and does go behind the object grouping. At present, we cannot give a fully satisfactory answer to this problem, but one possibility is that the space inside the cylinder is not treated as a unit by the navigational system. In this view, the cylinder consists of a large area with a trapezoid-like region cut out (the base of the trapezoid is an arc of the cylinder wall rather than a line segment) plus a small area between the objects and the nearby cylinder wall. It is possible to test this supposition indirectly by comparing the fraction of place cells with a field in the small region (4/17 or 0.235$)$ and the fraction of the cylinder made up by the small region ( $85 / 725$ or 0.117$)$. A binomial calculation shows that the probability of choosing $4 / 17$ cells with an a priori probability of 0.117 is $\sim 0.13$, suggesting that the small region is, indeed, represented separately. We conclude that the hypothesis that objects near the center are not used because of the complexity of the computations is not refuted by the clustered objects results.

An alternative explanation of the weak stimulus control exerted by central objects concerns the experiences available to laboratory rats. In the home cage, objects at the periphery (e.g., the food bin or water bottle) are stable, whereas central objects (e.g., other rats, sawdust) move around unpredictably. Accordingly, the rats may ignore the central objects because they have not had experience navigating by using stable central objects. An interesting experiment would be to raise rats with stable central objects; the place cells in such animals might be controlled by the objects, suggesting that the failure in the present case is not attributable to an inherent inability of the rat brain.

\section{Comparison of place cell results with behavioral data}

The experiments reported here are predicated on the idea that by studying place cells one is studying the neural implementation of a map-like representation of the environment. Because the map is presumed to allow the rat to solve certain spatial problems, it is important to ask whether or not animals are able to solve spatial problems by using stimuli similar to the objects used here. Clearly, it would be ideal if comparisons were made directly to the nature of spatial problem solving in a cylinder, with the same object set providing the only intentionally introduced cues. Such experiments having not yet been done, we relied on studies in rats and related species, which looked at how three-dimensional objects inside simply shaped environments affect the positional distribution of the rats' dwell time.

The elegant studies of Collett et al. (1986) show that gerbils are able to use objects similar to the ones used here to locate a food reward. The objects were one or more cylindrical landmarks, the relationship of which to the food and to each other (if more than one landmark were used) was kept constant during training. The gerbils proved capable of learning to find the food using such cues, but the task seemed to be very difficult; performance was still not fully reliable after 150 trials [Collett et al. (1986); see Gothard et al. (1996) for similar results in rats]. This result stands in great 
contrast to the rapid learning shown by rats in the hidden platform swimming task (Morris, 1981), where navigation is guided by distant stimuli.

The difficulty in learning to guide navigation relative to objects in the arena is quite parallel to our findings with place cells. In most cases there was no evidence of stimulus control in the objects-only conditions. In one case, however, the firing field of a cell was controlled consistently by the angular position of the objects. Interestingly, this was the last cell recorded from the rat, so a lot of time apparently was necessary before the rat began to use the objects to provide a reference direction. No other cells subsequently were recorded in this rat, but it is our strong prediction that once such control is achieved for a given cell it will be observed for simultaneously and subsequently recorded units. The notion is that the rat can, with difficulty, learn to use objects near the center of the apparatus to provide a reference direction and that the learning will be reflected in the control of all place cells, because the place cells form a map and are not merely a collection of independent units (Bostock et al., 1991; Knierim et al., 1995; Muller et al., 1996). We also note that the rapidity with which rats learn to guide locomotor behavior using distant cues (Morris, 1981) is parallel to the immediacy and consistency with which the white cue card controls the angular position of firing fields. Similar demonstrations of the greater efficacy of distant cues relative to local cues or path integration information are provided by a large number of studies (Suzuki et al., 1980; Téroni et al., 1987; Etienne et al., 1993; Alyan and Jander, 1994).

\section{Are object locations stored in the hippocampal map?}

Rats are able to store information about the identity and arrangement of objects in the environment. A rat that has explored a set of objects in a certain arrangement can be induced to reexplore the objects if a new object is substituted for one of the originals or if certain changes in the positional arrangement of familiar objects are made (Poucet et al., 1986; Thinus-Blanc et al., 1987, 1991). After hippocampal damage or temporary inactivation of the hippocampus, however, reexploration is observed only after individual items are swapped, but not when positional rearrangements are made (Poucet, 1989; Xavier et al., 1990; Save et al., 1992). Presumably, the hippocampus is not required for recognizing objects, but it is necessary for it to be possible to store object arrangements and the position of such arrangements with respect to the environment.

Why then do we see almost no tendency of place cell activity to reflect the existence and position of the objects? After all, the simple observation that the rats do not crash into the objects indicates that they influence navigation. We raise two possibilities.

The first is that the hippocampal portion of the navigational system represents only the layout of the environment. In this view the map does not contain an explicit representation of objects but, instead, serves as a substrate that allows the objects to be put in register with their locations in the environment in some other part of the brain, possibly in some part of the hippocampal formation other than Ammon's horn proper. This possibility follows directly from the data reported here and, in fact, is basically a restatement of our raw observations.

Proposing that the representation of object location is dissociated from the representation of the behavioral space is certainly convenient but has the disadvantage of failing to make any connection between our experiments and the related work of Gothard et al. (1996). We therefore consider a second possibility, namely that the lack of detecting effects of the objects near the center is attributable to our initial decision to focus on pyramidal cells with robust location-specific firing. It is possible that, had we recorded from all discriminable pyramidal cells, we would have found units that fired weakly but reliably when the rat was near any one of the objects. Such activity would remain in register with the object during spontaneous place cell field rotations in standard sessions and so would be stable in the laboratory frame even when the fields of the cell sample rotated. In addition, the postulated activity would rotate with object rotations even when the fields of the cell sample were not controlled by such rotations.

In short, the activity of the postulated "object-linked" units would appear in the objects-only conditions to be weak place cells under object control. This is exactly the sort of outcome that would be predicted from the exciting experiments of Gothard et al. (1996), who reported cell classes that fired in register with a box the animal left at the start of a trial and returned to at the end of a trial. In the case of Gothard et al. (1996), the box-related activity moved relative to the stable map, because the box was moved intentionally from place to place. In our case, the reference direction of the map jumps around unpredictably so that the postulated object-linked cells, although well behaved with respect to the objects, still would jump around relative to the map itself. In even closer parallel to the present experiments, Gothard et al. (1996) found cells for which the firing was in register with two objects that were moved from place to place in the environment; as suggested above, we might have seen similar cells tied to the central objects, although the majority of the cells was not controlled by the objects. To conclude, we imagine that no crisply firing object-linked cells were seen because the objects were behaviorally irrelevant. If the objects were, instead, necessary to solve a spatial problem, object-linked cells might be more active and therefore quite evident.

We conclude with a final speculation related to an interesting result of Biegler and Morris (1993). For the work reported in that paper, rats were trained to find food buried in sawdust a certain distance and in a certain direction from one of two demonstrably salient objects. For one group of rats the rewarded object $(\mathrm{L}+)$ was always in the same location relative to a large marker stimulus, a white sheet along one edge of a square, where the other walls were marked with black sheets. The unrewarded object $(\mathrm{L}-)$ also was at a fixed location relative to the sheet. For the other group, $\mathrm{L}+$ and $\mathrm{L}-$ were moved randomly from location to location relative to the white sheet, but the food was always at the same distance and direction relative to $\mathrm{L}+$.

Because rats can learn in the situation designed by Collett (Collett et al., 1986; Gothard et al., 1996), it is not surprising that the first group learned to find the food, and so, in fact, did the varied location group. The result of interest here, however, is the effect of removing the objects from the arena for either group. It was found that the rats exposed to the fixed location of $\mathrm{L}+\mathrm{did}$ not concentrate their search time near the expected location of the object nor of the food. Similarly, the rats exposed to the variable location of the object did not spend excess time in the portion of the apparatus in which $\mathrm{L}+$ or the food possibly could have been. Instead, both groups distributed their time approximately homogeneously, with some tendency to stay near the apparatus wall. In short, regardless of treatment, the rats acted as if they had no expectation of either the landmark locations or of the possibility of food reward in the apparatus. It was as if the chamber with and without the landmarks were two different places.

Preliminary results obtained in the course of the current work provide a tantalizing suggestion of what was happening in the 
Biegler and Morris experiment. In two cases a variant of the objects plus card experiment was done. The new manipulation was to rotate the cue card and leave the objects fixed relative to the laboratory frame. In other words, the relationship between the card and the objects was disrupted, just as the relationships between the sheet and the landmarks was disrupted by removing the landmarks in the Biegler and Morris experiment.

For both cells recorded under this circumstance, the positional firing pattern was altered in a manner indicative of a "complete remapping" (Quirk et al., 1990; Bostock et al., 1991). That is, the otherwise intact positional firing pattern did not merely rotate to a new angular position. Instead, the shape and radial position of the fields also obviously were changed. In previous work we have argued that, when firing fields of individual cells in two environments are transformed by more than rotation, the two environments have independent representations or maps. We now suggest that, if there are independent maps, any behavior emitted in one environment does not predict behavior in the second environment. In short, therefore, we think that the arena in the Biegler and Morris paper had two independent maps, one used when the landmarks were present and the other when they were removed. It is our prediction that, if place cell recordings were done in the Biegler and Morris situation, remappings would be seen for any rat that did not spend more time near the usual location of the landmarks plus food. For any rat that showed a preference to stay near the usual landmark location, we would predict that firing fields would be unchanged (even by a rotation) and that the maps of the environment with and without the landmarks would be the same.

\section{REFERENCES}

Alyan S, Jander R (1994) Short-range homing in the house mouse, Mus musculus: stages in the learning of directions. Anim Behav 48:285-298.

Batschelet E (1981) Circular statistics in biology. London: Academic.

Biegler R, Morris RGM (1993) Landmark stability is a prerequisite for spatial but not for discrimination learning. Nature 351:631-633.

Bostock E, Muller RU, Kubie JL (1991) Experience-dependent modifications of hippocampal place cell firing. Hippocampus 1:193-206.

Collett TS, Cartwright BA, Smith BA (1986) Landmark learning and visuo-spatial memories in gerbils. J Comp Physiol [A] 158:835-851.

Etienne AS, Joris-Lambert S, Reverdin B, Téroni E (1993) Learning to recalibrate the role of dead reckoning and visual cues in spatial navigation. Anim Learn Behav 21:266-280.

Gallistel CR (1990) The organization of learning. Cambridge, MA: MIT.

Gothard KM, Skaggs WE, Moore KM, McNaughton BL (1996) Binding of hippocampal CA1 neural activity to multiple reference frames in a landmark-based navigation task. J Neurosci 16:823-835.

Jung MW, McNaughton BL (1993) Spatial selectivity of unit activity in the hippocampal granular layer. Hippocampus 3:165-182.

Kelsey JE, Landry BA (1988) Medial septal lesions disrupt spatial mapping ability in rats. Behav Neurosci 102:289-293.

Knierim JJ, Kudrimoti HS, McNaughton BL (1995) Place cells, head direction cells, and the learning of landmark stability. J Neurosci 15:1648-1659.

Kubie JL (1984) A drivable bundle of microwires for collecting singleunit data from freely moving rats. Physiol Behav 32:115-118.

Morris RGM (1981) Spatial localization does not require the presence of local cues. Learn Motiv 12:239-260.

Morris RGM, Garrud P, Rawlins JNP, O’Keefe J (1982) Place navigation impaired in rats with hippocampal lesions. Nature 297:681-683.
Morris RGM, Schenk F, Tweedie F, Jarrard LE (1990) Ibotenate lesions of hippocampus and/or subiculum: dissociating components of allocentric spatial learning. Eur J Neurosci 2:1016-1028.

Muller RU, Kubie JL (1987) The effects of changes in the environment on the spatial firing of hippocampal complex spike cells. J Neurosci 7:1951-1968.

Muller RU, Kubie JL (1989) The firing of hippocampal place cells predicts the future position of freely moving rats. J Neurosci 9:4101-4110.

Muller RU, Kubie JL, Ranck JB (1987) Spatial firing patterns of hippocampal complex spike cells in a fixed environment. J Neurosci 7:1935-1950.

Muller RU, Stead M, Pach J (1996) The hippocampus as a cognitive graph. J Gen Physiol 107:663-694.

O'Keefe J, Conway DH (1978) Hippocampal place units in the freely moving rat: why they fire where they fire. Exp Brain Res 31:573-590.

O'Keefe J, Dostrovsky J (1971) The hippocampus as a spatial map. Preliminary evidence from unit activity in the freely moving rat. Brain Res 34:171-175.

O'Keefe J, Nadel L (1978) The hippocampus as a cognitive map. London: Clarendon.

O'Keefe J, Speakman A (1987) Single unit activity in the rat hippocampus during a spatial memory task. Exp Brain Res 68:1-27.

Paxinos G, Watson C (1986) The rat brain in stereotaxic coordinates. New York: Academic.

Poucet B (1989) Object exploration, habituation, and response to a spatial change in rats following septal or medial frontal cortical damage. Behav Neurosci 103:1009-1016.

Poucet B (1993) Spatial cognitive maps in animals: structure, contents, and functions. Psychol Rev 100:163-182.

Poucet B, Chapuis N, Durup M, Thinus-Blanc C (1986) A study of exploratory behavior as an index of spatial knowledge in hamsters. Anim Learn Behav 14:93-100.

Quirk GJ, Muller RU, Kubie JL (1990) The firing of hippocampal place cells in the dark depends on the rat's recent experience. J Neurosci 10:2008-2017.

Save E, Poucet B, Foreman N, Buhot MC (1992) Object exploration and reactions to spatial and nonspatial changes in hooded rats following damage to parietal cortex or dorsal hippocampus. Behav Neurosci 106:447-456.

Sharp PE, Blair HT, Etkin D, Tzanetos DB (1995) Influences of vestibular and visual motion information on the spatial firing patterns of hippocampal place cells. J Neurosci 15:173-189.

Skelton RW, McNamara RK (1992) Bilateral knife cuts to the perforant path disrupt spatial learning in the Morris water maze. Hippocampus 2:73-80.

Suzuki S, Augerinos G, Black AH (1980) Stimulus control of spatial behavior on the eight-arm maze in rats. Learn Motiv 11:1-8.

Taube JS, Kesslak JP, Cotman CW (1992) Lesions of the rat postsubiculum impair performance on spatial tasks. Behav Neural Biol 57:131-143.

Téroni E, Portenier V, Etienne AS (1987) Spatial orientation of the golden hamster in conditions of a conflicting location-based and routebased information. Behav Ecol Sociobiol 20:389-397.

Thinus-Blanc C, Bouzouba L, Chaix C, Chapuis N, Durup M, Poucet B (1987) A study of spatial parameters encoded during exploration in hamsters. J Exp Psychol Anim Behav Processes 13:418-427.

Thinus-Blanc C, Save E, Poucet B, Buhot MC (1991) The effects of reversible inactivation of hippocampus on exploratory activity and spatial memory. Hippocampus 1:363-369.

Thompson LT, Best PJ (1990) Long-term stability of the place-field activity of single units recorded from the dorsal hippocampus of freely behaving rats. Brain Res 509:299-308.

Xavier GF, Stein C, Bueno OFA (1990) Rats with hippocampal lesions do react to new stimuli but not to spatial changes of known stimuli. Behav Neural Biol 54:172-183. 